\title{
Informe del IV Congreso Nacional de Hospitales de Alta Especialidad y III Reunión Institucional de Auditorías Clínicas y Mejoría de la Calidad de la Atención Médica «La alta especialidad: una prioridad y un compromiso de equidad» 24-28 de septiembre de 2018, Oaxaca, México
}

\author{
Erick AzAmar-Cruz* \\ Director de Planeación, Enseñanza e Investigación; Hospital Regional de Alta Especialidad de Oaxaca, Oax., México
}

\section{PRESENTACIÓN}

La Comisión Coordinadora de Institutos Nacionales de Salud y Hospitales de Alta Especialidad y el Hospital Regional de Alta Especialidad de Oaxaca (HRAEO), con el soporte del Gobierno del estado de Oaxaca, teniendo como escenario el Centro Cultural y de Convenciones de Oaxaca, realizaron la cuarta edición del Congreso Nacional de Hospitales Regionales de Alta Especialidad y la III Reunión Institucional de Auditorías Clínicas y Mejoría de la Calidad de la Atención Médica. Ambos eventos se celebraron los días 24-28 de septiembre de 2018 y reunieron a destacados actores de la medicina de alta especialidad a nivel local, nacional e internacional, quienes expusieron temas de interés nacional y reflexiones alrededor de la necesidad de fortalecer la atención médica de alta especialidad y la mejora de la calidad de la atención médica hospitalaria, a través de la implementación de auditorías clínicas.

La organización del congreso se caracterizó por una importante agenda académica y cultural durante los cinco días -dos de precongreso y tres de congreso-, y su lema fue «Equidad en la atención médica.

En México, el derecho a la protección de la salud es un precepto plasmado con visión y alcance universal, y está basado en el reconocimiento de su necesidad, pero también en los principios de igualdad y equidad social.

Nuestra sociedad se caracteriza por la diversidad de concepciones del mundo y de los elementos que lo integran. En este sentido, la sociedad se enfrenta al reto de ser inclusiva, democrática y plural, pues el respeto y entendimiento de la visión del mundo desde la óptica del otro es la única vía que nos garantiza el avance y la permanencia de nuestra vida en comunidad.

Se entiende por inequidades en salud a las desigualdades evitables en materia de salud entre grupos de población de un mismo país o entre países. Esas inequidades son el resultado de desigualdades en el
Correspondencia:

*Erick Azamar-Cruz

E-mail: dirplaneacion@hraeoaxaca.gob.mx
Date of reception: $24-10-2018$

Date of acceptance: 12-11-2018

DOI: 10.24875/HMCM.M18000006 
seno de las sociedades y entre sociedades. Las condiciones sociales y económicas, y sus efectos en la vida de la población, determinan el riesgo de enfermar y las medidas que se adoptan para evitar que la población enferme o para tratarla.

En este sentido, "alcanzar la equidad en salud" significa que las personas puedan desarrollar su máximo potencial de salud independientemente de su posición social u otras circunstancias determinadas por factores sociales.

La equidad en salud implica que los recursos sean asignados según las necesidades.

\section{PRECONGRESO}

La actividad académica tuvo como preludio dos días de precongreso (24 y 25 de septiembre), en los que se dictaron 13 ponencias -4 médicas, 6 de enfermería, 1 de farmacia hospitalaria, 1 de laboratorio clínico y 1 administrativa-, con ponentes altamente capacitados del HRAEO, que compartieron experiencias y mejores prácticas resultado de los trabajos que realizan cotidianamente.

Algunos de los temas presentados fueron: abordaje del paciente quemado, manejo integral del delirio en pacientes en estado crítico, sepsis en la Unidad de Cuidados Intensivos ( $\mathrm{UCl}$ ), cirugía cardíaca, equipo de soporte para la prevención de las infecciones asociadas a la atención de la salud (IAAS), cuidados paliativos, manejo de heridas y estomas, gestión de la calidad en el laboratorio y presupuesto basado en resultados, entre otros, con los que se demostró la atención que se otorga a los pacientes. Se contó con un total de 365 asistentes, distribuidos en ambos días, posicionando al HRAEO como un referente en los programas de formación y actualización del personal de la salud en el estado de Oaxaca.

\section{CONGRESO}

Para el acto inaugural del congreso se contó con la presencia del titular de la Comisión Coordinadora de Institutos Nacionales de Salud y Hospitales de Alta Especialidad, el Dr. Guillermo Ruiz Palacios y Santos, con el director general de coordinación de los hospitales regionales de alta especialidad (HRAE), el Dr. Manuel de la Llata Romero, y con el Dr. Simón Kawa Karasik, director general de coordinación de los institutos nacionales de salud, así como con el Dr. Abelardo Meneses, director general del Instituto Nacional de Cancerología, y el Dr. Gerardo Pérez Bustamante, director general del HRAEO. En representación del gobernador constitucional del estado de Oaxaca, el ministro Alejandro Murat Hinojosa, asistió el ministro Donato Casas Escamilla, secretario de Salud Estatal, quien dirigió un mensaje a los congresistas y realizó el acto de inauguración de este magno evento el miércoles 26 de septiembre de 2018.

Durante los días 26-28 de septiembre se presentaron cinco conferencias magistrales, seis ponencias, dos mesas redondas con temas clínicos y de enseñanza, así como tres mesas redondas de auditorías clínicas, con la participación de profesionales adscritos a los HRAE, los institutos nacionales de salud, los hospitales federales de referencia, los servicios de atención psiquiátrica y la Universidad Nacional Autónoma de México, contando en su conjunto con 60 ponentes de extraordinario nivel.

Los temas abordados fueron: hígado graso no alcohólico, resistencia bacteriana, terapia endovascular neurológica, IAAS, cirugía cardiovascular y tamiz neonatal, enfermedades transmitidas por vector, enfermedades hematológicas, manejo multidisciplinario de la cirugía bariátrica, equidad en la alta especialidad y su futuro en México, enseñanza en los HRAE, historia de la medicina en Oaxaca y las auditorías clínicas en vías urinarias, salud mental y apego a Guía de Práctica Clínica (GPC), entre otros.

La difusión de las experiencias y las buenas prácticas realizadas en las instituciones nacionales de salud, además afianzadas por personal de alto nivel académico, fomentaron en los asistentes el interés, así como la necesidad de implementar nuevos proyectos en sus unidades de origen.

Durante estos 3 días de actividades académicas se contó con un total de 261 congresistas, entre ellos profesionales de la salud de diversas áreas e instituciones formadoras de recursos humanos del estado de Oaxaca, de institutos nacionales de salud y de otros hospitales de especialidad, y por supuesto de todos los HRAE. 


\section{SOCIOCULTURALES}

Para la realización del evento se contó con la ayuda del Gobierno del estado a través de la Oficina de Congresos y Convenciones y diversas dependencias, que llevó a cabo un coctel de bienvenida que tuvo verificativo en el segundo patio del Palacio Municipal de Oaxaca de Juárez -con la presencia de la Marimba del Estado-, un convite oaxaqueño con la participación de los trabajadores del HRAEO, directivos de la Comisión Coordinadora de Institutos Nacionales de Salud y Hospitales de Alta Especialidad (CCINSHAE) y de los HRAE, congresistas, visitantes y asistentes en general que, en un acto de hermandad y festejo, engalanaron con sus trajes regionales en la tradicional calenda oaxaqueña por las calles del centro histórico de la ciudad.

Por otro lado, se presentaron 14 stands de artesanías y alimentos típicos -algunos de familiares de pacientes del HRAEO-, así como de empresas farmacéuticas. Y el viernes 28 de septiembre, después del acto de clausura, presidido por los directores generales de los HRAE y el Dr. de la Llata Romero, se realizó la presentación de bailes de Guelaguetza. El evento se cerró con la degustación de platillos oaxaqueños.

\section{RESUMEN DE LÍNEAS DE INTERÉS ABORDADAS}

\section{Atención médica de alta especialidad}

La alta especialidad, que en nuestro país históricamente ha sido liderada por los institutos nacionales de salud y los hospitales regionales de referencia, se ha complementado con éxito en los últimos años con la consolidación de los HRAE, que han permitido descentralizar la atención médica altamente especializada, la formación de capital humano para la salud y la investigación hacia diversas regiones del país, mostrando resultados satisfactorios que apuntalan el objetivo supremo de coadyuvar en la mejora del estado de salud de la población mexicana.

Sin embargo, sigue vigente el reto demográfico y epidemiológico de la compleja agenda en salud pública, lo que crea la oportunidad de desarrollo, innovación y crecimiento de estos HRAE como elemento de fortalecimiento de las redes de servicios de salud estatales y nacional en favor de la salud de los mexicanos.

\section{Auditorías clínicas}

Se entiende por auditoría clínica la revisión de todos los aspectos de la actividad del cuidado clínico de los pacientes, realizada por profesionales de la salud (médico o no médico), cuya finalidad es mejorar la calidad. Durante la gestión actual se ha planteado la necesidad de implementarlas a partir del reconocimiento de las áreas de oportunidad de cada hospital.

Con la implementación de las auditoría clínicas se ha observado una mayor cumplimentación de la normativa, de acuerdo a las evaluaciones planteadas por los grupos multidisciplinarios, aunque también se siguen detectando áreas de oportunidad de mejora continua.

Se documentan diversas experiencias exitosas que plantean la importancia de continuar con el seguimiento e implementación de nuevas versiones de auditoría clínica en los diversos servicios hospitalarios.

\section{La formación de recursos humanos para la salud en México}

Es imperante la necesidad de contar con recursos humanos altamente capacitados para la salud, entre los cuales la formación de médicos especialistas sigue siendo una necesidad, por la demanda existente de los mismos en los múltiples hospitales del país, especialmente en los ubicados fuera de la megalópolis y en áreas de menor índice de desarrollo humano, con el objetivo de incrementar la cobertura de la atención médica especializada.

Este planteamiento permea la necesidad de contar con recursos presupuestales para la ampliación de plazas de residencias médicas y su posterior contratación por parte de las entidades federativas.

En promedio general, se encuentra ocupado el $72.6 \%$ de los campos clínicos disponibles, por lo que queda desocupado un $27.4 \%$ por falta de plazas de residentes. Además, las plazas de residentes 1 (R1) nacionales no han recuperado el nivel que tuvieron en 2012 y se mantienen la generación de conocimiento clínico y las rotaciones en vinculación con los Institutos Nacionales de Salud (140 en 2016 y 131 rotantes en 2017).

En los HRAE se cuenta con cursos avalados por las instituciones de educación superior que sólo pueden ofrecerse 
a extranjeros, por no contar con plazas nacionales, algunas de las cuales incluso se han suspendido, como es el caso de las de medicina crítica, geriatría e imagenología en el Hospital Regional de Alta Especialidad de la Península de Yucatán (HRAEPY), el HRAEO y el Hospital Regional de Alta Especialidad Ciudad Victoria (HRAEV).

Cada año, los HRAE se consolidan como una opción competitiva en la formación de los recursos humanos para la salud, lo que permite también la desconcentración de la formación del capital humano para la salud en las grandes ciudades.

\section{El futuro de la atención médica de alta especialidad en México}

El futuro inmediato deseable consiste en fomentar el ejercicio integral de la especialidad, a manera de antídoto contra:

- El abuso y la dependencia tecnológica.

- La visión fragmentada del paciente y su enfermedad.

- El deterioro de las capacidades clínicas.

- Los obstáculos de la burocracia institucional.

La visión de futuro se perfila en identificar las oportunidades de mejora que resultan del análisis histórico del estado actual de la atención médica desde la perspectiva de la división y cooperación en el trabajo médico.

Se perfilan las mejoras deseables en el futuro inmediato en términos de elevar las habilidades clínicas y desarrollar la atención médica centrada en el paciente en el marco de la tríada «servicio-docencia-investigación», como cúspide de la pirámide de la atención médica.

\section{El Hospital Regional de Alta Especialidad de Oaxaca, la piedra angular de la atención médica de alta especialidad en el estado de Oaxaca}

Dentro del sistema público de salud, el HRAEO es único en su tipo en el estado y la cúspide en la pirámide de los niveles de atención médica regional, siendo en muchas ocasiones la última alternativa para acceder a la atención médica que tiene la población sin derechohabiencia y de alta o muy alta marginación.
A pesar de ello, y después de casi 12 años de su creación, aún no cuenta con la certeza jurídica del predio que lo alberga a causa de diversas controversias políticas, como muchas que históricamente han afectado al estado, y ello se refleja en la imposibilidad de crecimiento, tanto en estructura como en servicios, compra de equipamiento y gestión de recursos humanos, entre otros aspectos. Durante el congreso se aprovechó para exponer tal situación a las autoridades nacionales y estatales con el objetivo de proponer una alternativa de solución viable y a corto plazo, esperando una respuesta favorable por parte del Gobierno estatal.

Cabe mencionar que en los últimos años, como consecuencia de este problema, se han dejado de recibir aportaciones de varios millones de pesos que hubieran posicionado aún más al hospital como la unidad de atención médica altamente especializada y resolutiva de la red, y hubiesen apuntalado el lema de la gestión como «una esperanza de vida».

\section{CONCLUSIONES}

Ante la encrucijada política que se avecina, es importante reconocer que, mientras que la atención primaria en salud no se vea fortalecida en el primer nivel de atención, los hospitales seguirán siendo los centros de curación por excelencia y el tercer nivel seguirá saturado con enfermedades de baja complejidad, en menoscabo de la calidad de la atención médica y, por consecuencia, de la oportunidad de atención para la población más vulnerable que realmente la requiera.

En consecuencia, es necesario el fortalecimiento del liderazgo institucional para una gobernanza en redes y una visión de hospitales de segundo y tercer nivel de atención en red que satisfagan las necesidades locales y regionales de salud, con trabajo colaborativo de cada entidad para atender así el continuo del proceso saludenfermedad en nuestro país.

Y para ello se requiere que los servicios de salud de cada estado sean la punta de la lanza en la gestión integral de los servicios médicos, a través de modelos de planeación estratégica de crecimiento, cobertura, carteras de servicios, formación de recursos humanos, formación de directivos, incentivos y cooperación técnica que atiendan nuestra realidad. 